\title{
The Effect of Stiffness and Curvature on the Haptic Identification of Surfaces
}

\author{
Vikram S. Chib \\ Sensory Motor Performance Program, \\ Laboratory for Intelligent Mechanical Systems, \\ Biomedical Engineering, Northwestern University \\ v-chib@northwestern.edu \\ Kevin M. Lynch \\ Laboratory for Intelligent Mechanical Systems, \\ Mechanical Engineering, Northwestern University \\ kmlynch@northwestern.edu
}

\begin{abstract}
We performed experiments to investigate how humans acquire an internal representation of virtual objects through the execution of reaching movements across the object surface. Subjects were instructed to make reaching movements between points lying on the boundary of a planar virtual surface of varying stiffness. Results suggest two types of internal representation: force perturbations and object boundaries. In the first case, a rectilinear hand movement is enforced by opposing the interaction forces. In the second case, the trajectory is conformed to the object boundary so as to reduce interaction forces. While this dichotomy is evident for very rigid and very soft objects, the likelihood of identifying a surface boundary depended, in a smooth and monotonic way, on the average force experienced in the first movements. This continuum of interactive behaviors suggests that the nervous system uses a weighted combination of two control strategies, one generating a compensatory response, the other a compliant motion.
\end{abstract}

\section{Introduction}

A number of studies have been performed to understand the perception of shape through active touch. Kappers and colleagues found that humans are capable of learning and distinguishing slight differences in the curvature of various surfaces [1]. Investigation of actively touched curved surfaces has shown that adaptation and after-effects are present following haptic exploration [2]. These haptic aftereffects are manifested as flat surfaces being judged as convex following the touching of a concave surface,

\author{
James L. Patton \\ Sensory Motor Performance Program, \\ Biomedical Engineering, Northwestern University \\ j-patton@northwestern.edu
}

Ferdinando A. Mussa-Ivaldi

Sensory Motor Performance Program, Biomedical Engineering, Department of Physiology, Northwestern University sandro@northwestern.edu

and flat surfaces being judged as concave following the touching of a convex surface. Haptic aftereffects increase with the time of contact with the curved surface, and decrease with the time elapsed between the touching of two different surfaces [3].

The degree of rigidity, or stiffness, of an object is critically important for manipulation. Psychophysical studies have been performed to determine thresholds for stiffness discrimination. Using a contralateral limb matching procedure, in which subjects adjusted the stiffness of a motor connected to one arm until it was perceived to be the same as that connected to the other arm, Jones and Hunter determined that the sensitivity of stiffness discrimination was much worse than would be expected by combining the sensitivities for force and displacement discrimination [4].

Rigid objects, such as walls and table tops, are characterized by high impedance boundaries. Accordingly, when the hand comes in contact with such an object there is minimal or negligible penetration inside the boundary. Other objects, such as pillows and computer keyboards, respond to applied forces with larger displacements. In mechanical terms, these different behaviors are captured by describing objects as fields of positiondependent forces. In this work we investigate interactions between the hand and objects as interactions of the hand with external force fields.

Experiments presented in this paper are an extension of previous work [10] which found that subjects' internal representation of virtual objects may be characterized by two distinct categories through a continuum of force fields: force perturbations and object boundaries. This paper 
explores how perceptions of virtual objects are effected by their stiffness and curvature. In these experiments, subjects execute hand movements while holding a device that rendered planar virtual objects with variable stiffness and radius.

\section{Materials and Methods}

\subsection{Experimental Apparatus}

All experiments were performed using a two degree of freedom planar manipulandum as seen in Figure 1. Subjects made goal-directed movements in the plane of the manipulandum while grasping its handle. The manipulandum is similar to those previously described $[5,11]$. It is instrumented with positional encoders which record angular position of its links. Position and velocity of the manipulandum handle are computed from these encoder signals. These signals are sampled continually at a frequency of $100 \mathrm{~Hz}$. The manipulandum is also equipped with two torque motors that generated the force fields corresponding to the virtual object. Endpoint forces are acquired using a six degree of freedom load cell fixed to the handle of the robot.

\subsection{Virtual Object}

The force fields experienced by subjects were defined by the following formula:

$$
F(r)=\left\{\begin{array}{cc}
K(R-r)+B \dot{r} & r \leq R \\
0 & r>R
\end{array}\right\}
$$

This formulation defines a circular, elastic, virtual disk of radius $R$ centered at $O$. The interface force produced when contacting the virtual disk was proportional to the stiffness of the disk $K$ and the displacement into the boundary $r$. A component of damping $B$ was incorporated into this formulation to alleviate instabilities encountered at higher stiffnesses. This is a technique commonly used when programming virtual surfaces [12].

\subsection{Experimental Protocol}

Subjects made goal directed reaching movements from a start target to a goal target. During a given trial a target was projected onto the subject's workspace and the subject was asked to make one continuous movement to place a cursor registered to the manipulandum handle within the target, while achieving a desired maximum velocity. The next target appeared after the subject held the cursor at the prior target for one second. Subjects were given feedback if they moved faster or slower than the desired maximum velocity. These feedback cues allowed subjects to achieve a consistent maximum speed of movement of $0.40 \mathrm{~m} / \mathrm{s}$.

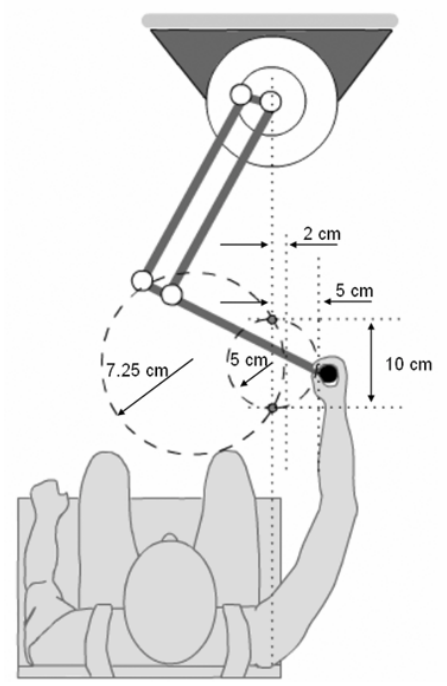

Figure 1. Schematic of manipulandum and dimensions of virtual surfaces.

Prior to the introduction of force fields subjects practiced making point to point movements under the required velocity constraints, in the absence of a virtual object, for 60 movements. In order to assess the typical performance of the subject, undisturbed in free space, objects were not introduced during this baseline unperturbed phase. This phase of the experiment allowed subjects to familiarize themselves with the dynamics of the manipulandum.

Following the baseline unperturbed phase virtual objects were presented to the subject. Subjects were only given a haptic presentation of the virtual object; visual information regarding the geometry of the object was not presented. The dimensions of the virtual object can be seen in Figure 1. A testing phase consisted of the subject moving between targets located on the boundary of the virtual object. Subjects made 100 reaching movements between the presented start and goal positions. The first 50 movements of a testing phase served as a learning period for the subject to acquire information about the virtual surface. During the final 50 movements of the phase catch trials were introduced pseudorandomly for $12.5 \%$ of the movements. These catch trials, movements during which no force field was present, were introduced to reveal any adaptations of the feedforward motor command that may have occurred after training with the virtual object. 
After completion of the phase consisting of 100 movements with the virtual object, a "wash-out" phase consisting of 50 movements in a null field was introduced. This phase allowed for de-adaptation and unlearning of the field encountered during the previous phase. Six different stiffness levels were tested $(200,400,800,1200,1600,2000 \mathrm{~N} / \mathrm{m})$. The stiffness levels were presented in order of increasing magnitude. One group of subjects (Low-Curvature Group) was exposed to the various stiffness levels with a virtual disk of curvature $14 \mathrm{~m}^{-1}(R=7.25 \mathrm{~cm})$, while a second group (High-Curvature Group) was exposed to the same stiffness levels with a virtual disk of curvature $20 \mathrm{~m}^{-1}(R=5 \mathrm{~cm})$.

\subsection{Trajectory Analysis}

Two measures were used to quantify subjects' response to virtual objects and their subsequent learning.

\subsubsection{Area Reaching Deviation}

The measure of area reaching deviation (ARD) was used to evaluate a subjects' deviation from a straight line path, due to the exposure of a virtual surface,. This measure was defined as the signed area between the trial trajectory and a reference straight line path between the start and goal positions. Area reaching deviation is the spatial average of the lateral deviation away from the reference straight line trajectory. Trajectories to the right of the reference straight line trajectory were given positive weight, while those to the left were given a negative weight.

$$
A=\int_{y_{i}}^{y_{f}} x d y
$$

\subsubsection{Interface Force}

The measure of interface force was used to evaluate the forces imparted by the virtual object during subjects' movements. These force samples were integrated over the duration of the movement to acquire a resulting force cost (Equation 2.4) for an entire reaching movement.

$$
C=\frac{\int_{t_{i}}^{t_{f}} F(r, t) d t}{t_{f}-t_{i}}
$$

\subsubsection{Psychometric Function}

A common means of quantifying a subject's performance of a psychophysical task is the fitting of a psychometric function. The psychometric function relates an observer's performance of a psychophysical task to some physical aspect of stimulus. For these experiments the performance metric used was the sign of the ARD. A twoalternative paradigm was implemented for the catch trials performed at each stiffness level. Catch trials having a negative ARD were classified as perception of a field while those having a positive ARD were classified as perception of an object boundary or surface. Subjects' results were compiled into a single group measure for each stiffness level. This measure was expressed as the proportion of positive surface responses at each stiffness level.

\section{Results and Discussion}

Selected movement trajectories for a subject in the Low-Curvature Group, with different field stiffness and at different stages of learning (early exposure and late exposure) are shown in Figure 2. The time course of movements during the early exposure phase can be divided into two segments. During the first segment, the hand was driven off course by the field and forced away from a straightline trajectory. During the second segment of movement, after the force field of the virtual object had caused the hand to veer off course from the target, subjects made a second movement back towards the target. At low stiffness values $(200 \mathrm{~N} / \mathrm{m}$ and $400 \mathrm{~N} / \mathrm{m}$ ), after adaptation, subjects produced straight-line movements through the field (Figure 2). Repeated practice of movements with high-stiffness virtual surfaces $(k=800,1200,1600,2000 \mathrm{~N} / \mathrm{m})$ resulted in a markedly different adaptation. Unlike adaptation to low stiffness fields, adaptation to high stiffness fields did not result in subjects recovering straight-line movements. Instead subjects produced movements that followed the curvature profile of the virtual surface.

These qualitative observations of subjects' adaptations to virtual objects of varying stiffness are quantified for all subjects in Figure 3a. As previously described, with low stiffness values $(200$ $\mathrm{N} / \mathrm{m}$ and $400 \mathrm{~N} / \mathrm{m}$ ), after adaptation subjects produced straight-line movements through the field (Figure 2). This result was captured by the area reaching deviation (ARD) before and at the end of practice. ARD for virtual surfaces of 200 and 400 $\mathrm{N} / \mathrm{m}$ were significantly different $(\mathrm{p}<0.05$ ) (Figure 3a). At the high stiffness levels ARD was not significantly reduced after learning. The data in Figure $3 \mathrm{a}$ demonstrate that the measure of ARD increases as the stiffness level increases. Thus, as stiffness is increased, subjects began to produce trajectories that conformed to the boundary of the virtual object, as opposed to actively counteracting the forces generated by the virtual object. 


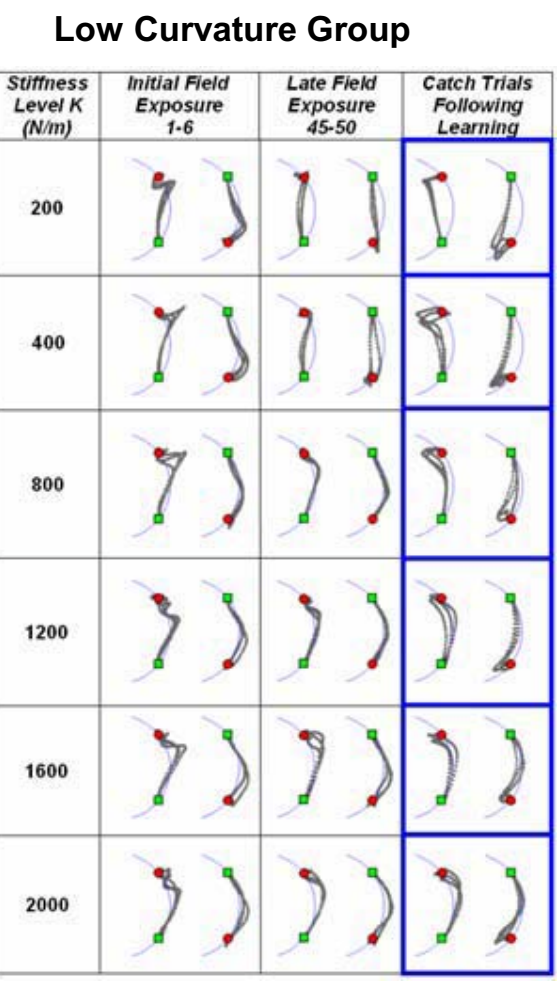

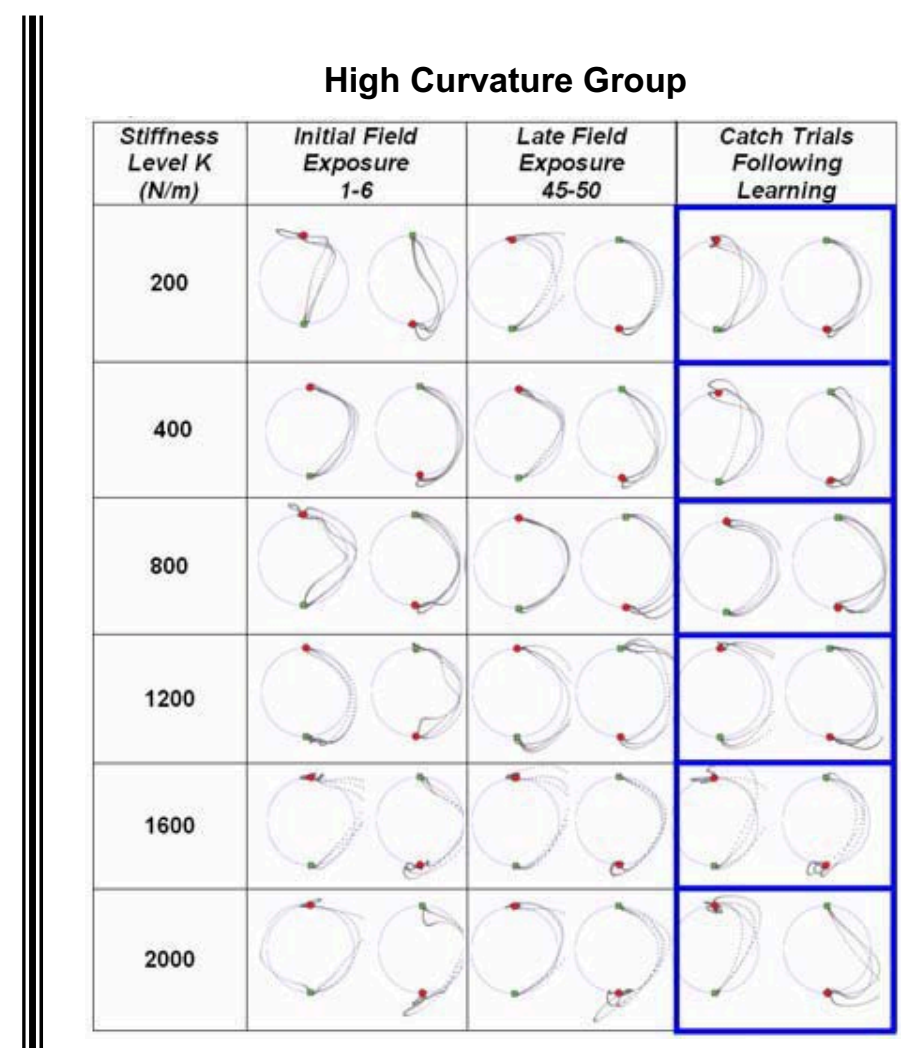

Figure 2. Trajectories from various stages of adaption for typical subjects. Green squares represent the start position, red circles represent the goal position.

Low Curvature Group

a.

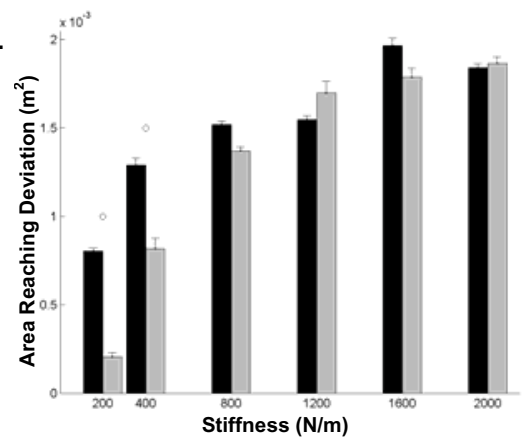

b.



Before Learning

\section{High Curvature Group}

c.

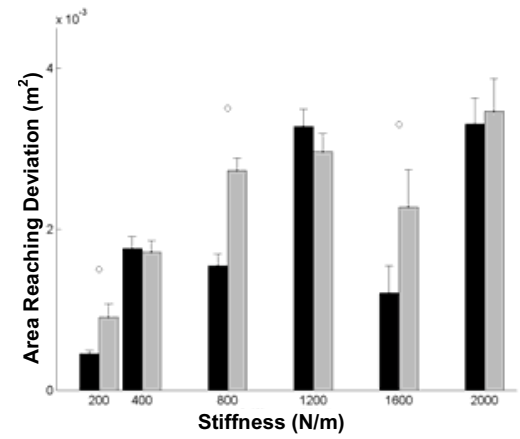

d.

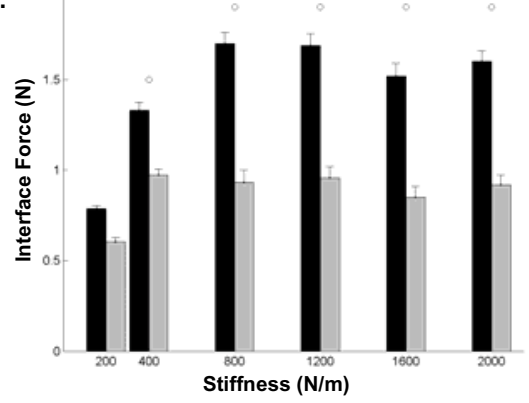

After Learning

$\bigcirc p<0.05$ SE

Figure 3. Learning data for both groups. Measures shown are an average of the first and last five trials averaged across all subjects. (a) Area reaching deviation. (b) Interface Force. 
A unifying feature amongst the adapted movements is the average interface force. Through the continuum of stiffness levels subjects tended to produce similar average interface forces after adaptation (Figure 3b). A two-factor ANOVA did not find a significant difference amongst average interface force for the six stiffness levels, for subjects in the low-curvature group after adaptation had occurred $\left(\mathrm{F}_{6,5}=0.187 ; \mathrm{p}=0.966\right)$.

After-effects from adaptation to the low-stiffness field were observed during catch trials at the end of training. These after-effects are mirror images of the responses to the initial field exposure (Figure 2). This suggests that in the presence of low-stiffness position-dependent force fields, subjects adapted by developing an internal representation of the field. The internal representation predicted and canceled the forces of the virtual object. At higher stiffness levels this was not the case. Once a stiffness threshold was exceeded, subjects' after-effects appeared in the direction of the applied forces and following the profile of the virtual surface. The amount by which the after-effect's appeared in the direction of the applied forces of the virtual surface and away from a straight line trajectory increased as the stiffness of the surface increased.

\subsection{Effect of surface curvature}

For the High-Curvature Group, we did not observe the force field adaptation with recovery of straight line movements, at low stiffness values. Instead, after training with the low stiffness surface, subjects exhibited movements which complied with and conformed to the circular disk boundary (Figure 2). This result was manifested in subjects increasing ARD at lower stiffness levels after learning (Figure 3c). Furthermore, after-effects from this higher curvature level remained positive and gradually increased across all stiffness levels indicating that subjects produced after-effects in the direction of the applied forces and approximately conforming to the profile of the virtual boundary. The High-Curvature group showed a monotonic increase of ARD for catch trials (Figure 4) as the stiffness level of the virtual surface increased.

A common feature between the adaptations to fields of different curvatures was the constancy of average interface force experienced after learning. Results showed that in the case of the higher curvature surface, subjects again achieved an invariant level of interface force (Figure 3d). While the level of force was larger than that found for the Low-Curvature Group, it remained relatively constant across all stiffness levels. A two factor ANOVA without replication did not find a significant difference amongst the six stiffness levels $\left(F_{6,5}=1.272 ; p=0.307\right)$ after learning.

\subsection{Modulation of average interface force}

Results showed that the unifying theme across these stiffness and curvature levels was subjects' tendency to generate a constant level of interface force regardless of object mechanics. A regression of interface force before learning versus ARD after

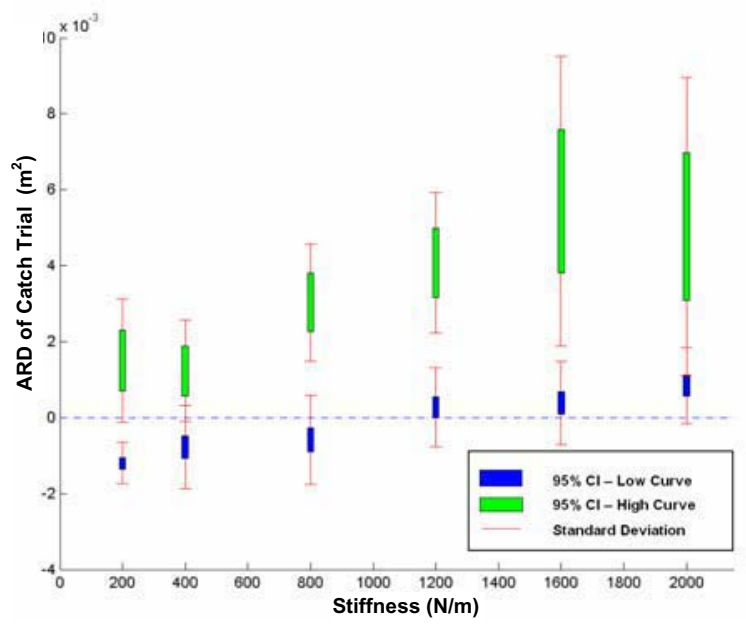

Figure 4. Area reaching deviation for catch trials of subjects moving in contact with a low curvature and high curvature surfaces.

learning (Figure 5a) showed very similar trends in both curvature groups. This suggests the presence of a common adaptation strategy for the Low-Curvature and High-Curvature Groups, whose outcome depends on the amount of interface force experienced in the early interactions with the field/surface. Accordingly, the level of initial interface force experienced may be the key factor in subjects' perception of a field as a surface. A gradual trend was seen between subjects' level of interface force before learning and their probability of perceiving a surface (Figure 5b). For this psychometric fitting perception of a surface was classified as the threshold of pre-adaptation interface force at which subjects began to perceive surfaces greater than chance occurs at approximately 1.0 Newton. The fact that interface force, rather than stiffness or curvature, is the best predictor of the final classification suggests that the feedforward command plays a central role in haptic perception.

\section{Conclusions}

Findings demonstrate the existence of both compensatory and compliant responses occurring at 

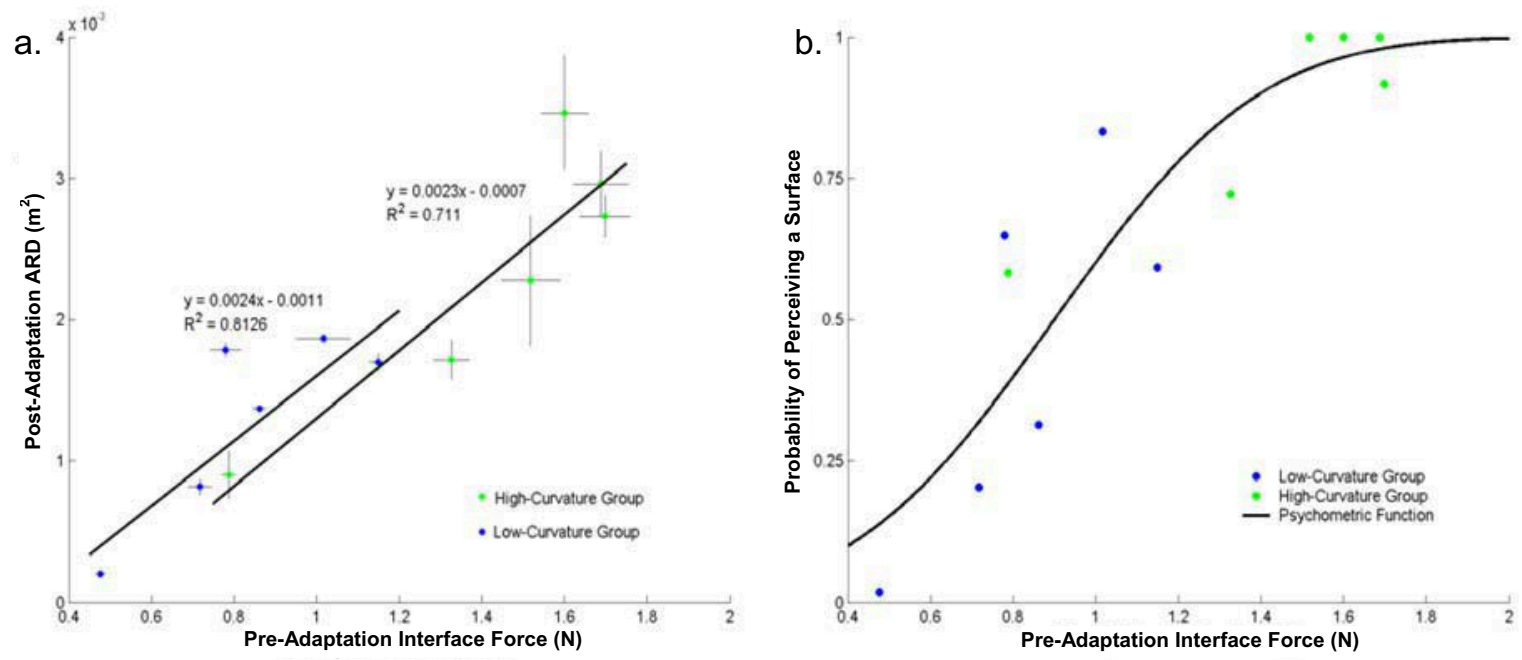

Figure 5. Unified curvature results. (a) ARD after learning versus interface force before learning for both LowCurvature and High-Curvature Groups. (b) A unified psychometric function of perception of surfaces for an average interface force before learning.

different values of stiffness. The lowest values of stiffness lead to compensatory responses in which a free space trajectory is recovered, while the highest values lead to compliant responses. A smooth transition from compensation to compliance appeared across a continuum of stiffness levels. Objects with different stiffness induced, as expected, different levels of interaction forces. However, a striking effect of practice was a strong reduction in these interaction forces to a constant level. Taken together, these studies reveal a mechanism of adaptation that may subserve the implicit learning of object shapes through repeated haptic interactions. The curvature and stiffness of the boundary appear to be important parameters that may either inhibit or promote such learning processes by establishing a critical level of interaction force between hand and object: compensatory responses are predominant when the pre-adaptation interaction force is below this critical level and compliant responses are predominant when the interaction force exceeds this level.

\section{Acknowledgements}

This work was supported by NIH Grant F31NS049795, NIH Grant NS35673, and NSF Grant IIS0082957. All human testing was approved by the Northwestern University Institutional Review Board.

\section{References}

[1] A. Kappers, J. Koenderink, and I. Lichtenegger, "Haptic identification of curved surfaces," Perception \& Psychophysics, vol. 56, pp. 53-61, 1994.

[2] I. Vogels, A. Kappers, and J. Koenderink, "Haptic aftereffect of curved surfaces," Perception, vol. 25, pp. 109-119, 1996.
[3] I. Vogels, A. Kappers, and J. Koenderink, "Haptic after-effect of successively touched curved surfaces," Acta Psychologica, vol. 106, pp. 247-263, 2001.

[4] L. Jones and I. Hunter, "A perceptual analysis of stiffness," Experimental Brain Research, vol. 79, pp. 150-156, 1990.

[5] R. Shadmehr and F. Mussa-Ivaldi, "Adaptive Representation of Dynamics During Learning of a Motor Task," Journal of Neuroscience, vol. 14, pp. 3208-3224, 1994.

[6] Y. Matsuoka, "Models of Generalization in Motor Control," in Department of Electrical Engineering and Computer Science. Cambridge: Massachusetts Institute of Technolgoy, 1998, pp. 215.

[7] F. Gandolfo, F. Mussa-Ivaldi, and E. Bizzi, "Motor learning by field approximation," Proceedings of the National Academy of Science USA, vol. 93, pp. 38433846, 1996.

[8] K. Thoroughman and R. Shadmehr, "Learning of action through adaptive combination of motor primitives," Nature, vol. 407, pp. 742-747, 2000.

[9] T. Flash and I. Gurevich, "Arm stiffness and movement adaptation to external loads," presented at Annual Conference on Engineering in Medicince and Biology, 1992.

[10] V. Chib, J. Patton, K. Lynch, and F. Mussa-Ivaldi, "Haptic Discrimination of Perturbing Fields and Object Boundaries," presented at International Symposium on Haptic Interfaces for Virtual Environments and Teleoperator Systems, Chicago, IL, USA, 2004.

[11] F. Mussa-Ivaldi and E. Bizzi, "Motor learning through the combination of primitives," Philisophical Transactions of the Royal Society of London, vol. 355, pp. 1755-1769, 1999.

[12] J. Colgate and J. Brown, "Factors Affecting the ZWidth of a Haptic Display," presented at IEEE International Conference on Robotics \& Automation, San Diego, CA, 1994. 